\title{
Patients Left Without Being Seen in the Emergency Department - A Retrospective Observational Patient Record Study
}

Markus Holmberg ( $\sim$ markus.holmberg@khshp.fi )

Kanta-Häme Central Hospital

\section{Tapio Innamaa}

Kanta-Häme Central Hospital

Ville Hällberg

Kanta-Häme Central Hospital

Timo Lukkarinen

Kanta-Häme Central Hospital

Ari Palomäki

Kanta-Häme Central Hospital

\section{Research Article}

Keywords: LWBS, left without being seen, emergency department, emergency medicine

Posted Date: July 28th, 2021

DOI: https://doi.org/10.21203/rs.3.rs-744447/v1

License: (a) (i) This work is licensed under a Creative Commons Attribution 4.0 International License. Read Full License 


\section{Abstract}

\section{Background}

The share of patients leaving the emergency department without being seen by a physician (LWBS) is an important efficacy marker of emergency departments around the world. It has not however, been of special research interest in the Nordic countries. In this study, we assessed the LWBS rate in Kanta-Häme Central Hospital, in Hämeenlinna, Finland. Secondarily, we gathered information of LWBS patients along with all ED patients.

\section{Methods}

In this retrospective observational patient record study, our primary aim was to assess the LWBS rate of all patient visits $(n=41,631)$ over a 12-month period in a mid-sized Finnish secondary hospital ED. Alongside, we gathered specific information, such as age, gender, time of day and year for attendance, reason for attendance, return to healthcare and mortality after 7 and 30 days and after one year to show characteristics of LWBS patients. We present data and hourly distribution of the LWBS patients against all patient visits to the ED.

\section{Results}

A total of 305 LWBS cases were identified, leading to an LWBS rate of $0.73 \%$. Most LWBS took place during afternoon hours, when the ED was most crowded and waiting times were longest. Among LWBS patients, minor traumas and non-specific pain were the most common reasons for attending. Mortality among LWBS patients was naught $(0.0 \%)$ over a one-year follow-up.

\section{Conclusions}

The LWBS rate in the Kanta-Häme Central Hospital ED was low. Higher LWBS rate coincided with rush hours and longer waiting times. The proportion of minor traumas was greater than in some earlier studies. These results support the implementation of a fast track line for minor traumas in the ED.

\section{Background}

The share of patients leaving the emergency department (ED) without being seen by a physician (LWBS) is considered one of the main efficacy markers of EDs worldwide [1, 2]. It has not, however, been of special research interest in the Nordic countries. The main reasons for LWBS are overcrowding in the ED and long waiting times $[1,3,4]$. LWBS patients are considered a consequence of overcrowded or poorly functioning EDs and may ultimately, although rarely, lead to serious harm to such patients [5].

Our primary aim in this study was to assess the LWBS rate of the Kanta-Häme Central Hospital (KHCH), a middle-sized secondary teaching hospital in Finland. The secondary aims were to study the profiles of LWBS patients together with seasonal and temporal variations. Further, we attempted to identify factors 
which, if remedied, would help us to improve the efficacy of the ED. This study is a part of the research and reform projects conducted to systematically improve the processes in $\mathrm{KHCH} E D$, such as the reorganization of our stroke treatment protocol $[6,7,8,9,10]$.

\section{Methods}

The data consist of all patients attending the ED of KHCH over a 12-month period $(n=41,631)$. We evaluated LWBS patients from patient records. This identification was made from the so-called $E$-book, where patients checked in by a triage nurse and leaving without being seen by a doctor are registered. In our ED, every patient entering is triaged. Reasons for attending were collected from patient records as written by the triage nurse. We divided these into 14 categories based on the study by Carron et al. [4]. The date, day of the week, time of arrival, time of departure, age, gender, possible return to our hospital or other health care unit within seven days and 7 to 30-day mortality were also collected for statistical analysis. No triage category was obtainable retrospectively during the time of data collection.

The absolute number and rate of LWBS patients with seasonal and temporal variations were calculated. Further, all patient visits to the ED and their absolute waiting times per hour together with mean and median waiting times with $95 \%$ confidence intervals were collected.

The study was a retrospective patient record study thus requiring no consent from patients. No ethical statement on the study was required the ethics committee of Tampere University Hospital according to Finnish legislation (Medical Research Act 488/1999). The study was given permission from KHCH (n:o KHSHP/174/13.00.01/2021).

\section{Results}

We identified a total of 305 LWBS visits, $99.0 \%$ of whose arrival times were available $(n=302)$. The 305 visits consisted of 299 individual patients, six patients made two separate visits ending in LWBS. Time of departure was available for $55.4 \%(n=169)$ of visits and reason for attending for $99.3 \%(n=303)$. The characteristics of LWBS patients compared to those of all ED visits are shown in Table 1. Of these patients, $28.5 \%$ were less than 18 years of age. When comparing LWBS patients to the normal age distribution of all our patients, we can see an overrepresentation of age groups $\leq 35$ years of age and male gender. 
Table 1

Distributions of LWBS patients during the study period.

\begin{tabular}{|lllll|}
\hline & & $\begin{array}{l}\text { Number of } \\
\text { patients (N) }\end{array}$ & $\begin{array}{l}\text { Distribution of LWBS } \\
\text { patients (\%) }\end{array}$ & $\begin{array}{l}\text { LWBS / all ED } \\
\text { patients (\%) }\end{array}$ \\
\hline $\begin{array}{l}\text { All ED } \\
\text { patients }\end{array}$ & 41631 & & \\
\hline - Gender & Male & 19934 & & 0.79 \\
\hline & Female & 21697 & & 0.68 \\
\hline $\begin{array}{l}\text { LWBS } \\
\text { patients }\end{array}$ & 305 & 51.8 & 0.73 \\
\hline - Gender & Male & 158 & 48.2 & \\
\hline & Female & 147 & 28.5 & 1.32 \\
\hline - Age & 87 & 17.0 & 1.25 \\
\hline $18-25$ & 52 & 15.7 & 1.12 \\
\hline $26-35$ & 48 & 9.5 & 0.72 \\
\hline $36-45$ & 29 & 11.1 & 0.72 \\
\hline $46-55$ & 34 & 8.5 & 0.45 \\
\hline $56-65$ & 26 & 7.5 & 0.38 \\
\hline $66-75$ & 23 & 2.0 & 0.06 \\
\hline$\geq 76$ & 6 & & \\
\hline $\begin{array}{l}\text { Distributions of LWBS patients are presented as numbers (N) and percentages (\%) according to } \\
\text { gender and age groups. LWBS rate (\%) in different age groups and genders is shown in the final } \\
\text { column. ED = Emergency Department; LWBS = Left Without Being Seen. }\end{array}$ \\
\hline
\end{tabular}

The overall LWBS rate was $0.73 \%$. A significant monthly fluctuation in LWBS rate was seen peaking in June at $1.40 \%$ (Fig. 1).

Most LWBS took place during daytime. The number LWBS patients reflected well with the total number of patients in the ED (Fig. 2a). The waiting time $(n=166)$ before LWBS was 75.5 minutes (median) and 89.5 minutes (mean; 95\% confidence interval 79.3-99.7 minutes). For the whole patient population, the average mean waiting time before seeing the doctor varied between 48 and 92 minutes according to the time of the day, the shortest being at nighttime and the longest during the afternoon hours (Fig. 2b). A slight increase in waiting time to doctor can be seen at seven o'clock in the morning, which is most likely attributed to shift changes of doctors and nurses between seven and eight o'clock.

The most common cause for attendance ending in LWBS was minor trauma $(29.4 \%)$ and the second most common cause was non-specific pain (12.9\%) (Table 2). Adverse effects of alcohol and/or drugs 
were the main reasons for attending in only $3.6 \%$ of LWBS cases but had some effect on a larger proportion of cases. There were only 214 patients whose native language was not Finnish or Swedish, the native languages in Finland ( $0.5 \%$ of all ED visitors). Their share in the LWBS group did not differ from that in the total population of the catchment area.

Table 2

LWBS patients' reasons for attending the ED in 14 categories.

\begin{tabular}{|lll|}
\hline Reason for attending (categorized) & N & $\%$ \\
\hline Trauma & 89 & 29.4 \\
\hline Non-specific pain & 39 & 12.9 \\
\hline Ear nose throat complaints & 31 & 10.2 \\
\hline Infections & 22 & 7.3 \\
\hline Abdominal complaints & 21 & 6.9 \\
\hline Urological and/or gynaecological complaints & 18 & 5.9 \\
\hline Eye / ophthalmological complaints & 17 & 5.6 \\
\hline Neurological complaints & 15 & 5.0 \\
\hline Psychiatric problems & 13 & 4.3 \\
\hline Alcohol and drug-related problems & 11 & 3.6 \\
\hline Cardiovascular complaints & 9 & 3.0 \\
\hline Pulmonary complaints & 8 & 2.6 \\
\hline Skin problems and allergies & 7 & 2.3 \\
\hline Endocrinological problems & 3 & 1.0 \\
\hline Total & 303 & 100.0 \\
\hline $\begin{array}{l}\text { Reasons of LWBS patients for attending the ED are categorized according to the study by Carron et al. } \\
\text { [4] with slight modifications. Abbreviations, see Table 1. }\end{array}$ & \\
\hline
\end{tabular}

Of LWBS cases, $33(10.8 \%)$ returned to the ED or some other health care provider during the following seven days after LWBS and only two more (0.66\%) between 7 and 30 days. None of the LWBS patients ( 0 $\%)$ died within one year of follow-up.

\section{Discussion}

After the implementation of the independent medical speciality of Emergency Medicine in Finland [11], we have systematically been analyzing and developing our processes and clinical environments $[6,7,8,12$, 13]. The LWBS rate in our study was low compared to those reported in other studies $[14,15,16,17,18$, 
$19,20]$. Rush hours and longer waiting times coincided with larger numbers of LWBS, even though the total rate was low. Younger patients and patients with minor traumas formed the largest LWBS groups in our study.

Waiting times and rush in the ED are well studied factors associated with increased LWBS rates worldwide $[4,14,16,18,19,20,21,22]$. In this study we noticed that when waiting times before being seen by a physician exceeded 75 minutes, mostly during the afternoon hours with more patients, the LWBS rate increased. In some studies, nighttime was a risk factor for LWBS, but this was not apparent in the present study $[15,17,23]$.

In our study hospital the proportion of minor traumas among LWBS patients as the reason for attending was rather high (29.4\%), unlike in the studies by Grosgurin et al. (15.0\%) and Parekh et al. (18.3\%) [15, 16]. Younger age groups with a slight male preponderance were over-represented in LWBS in our study, which has also been reported in other studies $[14,15,24]$.

In some studies, non-native language speaking background or non-compensable coverage status have been a strong predictor for LWBS $[25,26,27]$. In this study, however, patients whose native language was not Finnish were not overrepresented in LWBS. In Finland, all patients are covered by a municipal tax and the visit fees of EDs are low. Due to the retrospective nature of this study, visit fees as a cause for LWBS were not specifically elicited. However, according to the feedback query system of our ED, visit fees have been a cause of complaints only in sporadic cases. Symptom relief was probably also in some of our patients associated with LWBS as also in some earlier studies [28, 29].

A strength in this study was the large number of total patients studied. We believe that LWBS patients were comprehensively included in the study, since our health records (including the E-book) are well kept and also linked to the municipal payment transactions. The reason for attendance could also be reliably ascertained since on entering the ED every patient was seen by an experienced triage nurse. This yields a trustworthy estimate of the true proportions of different causes for LWBS patients attending the ED. Further, mortality is based on national records in Finland, which are comprehensive and reliable [30].

The limitations of this single centre study are mainly due to the study design, as it is retrospective. In a prospective study, we would have been able to specify the reasons behind LWBS and waiting times even more accurately than now. On the other hand, patients admitted to a prospective study might be less inclined to leave before seeing a doctor, thus causing a bias. Not all health records in Finland have so far been fully connected to the national database. Therefore, we have no means of knowing about every visit made to private sector healthcare facilities.

Reducing the LWBS rate is an important factor in present-day goal-directed ED management. This improves both patient satisfaction and safety as, although complications are rare, these may occur. Applying lean healthcare interventions or specific fast-track lines for patients improves LWBS rates and throughput in the $\operatorname{ED}[31,32,33]$. These suggestions may further improve our results, since the proportion of children and minor traumas suitable for the fast tract policy was high in our LWBS population. To 
tackle the LWBS problem, we have already implemented an "ED lobby project" aiming to reduce waiting times for ambulatory patients and applying the principles outlined earlier [13].

\section{Conclusions}

The LWBS rate in our ED was low, only $0.73 \%$. The reasons for attending among LWBS patients differed slightly from those reported in other studies as minor traumas were over-represented. Our results support the implementation of a fast-track line for minor traumas, which could further improve the LWBS rate. Since waiting times to physician contact were more than 75 minutes, especially during the afternoon hours, adequate targeting of resources could further help to reduce the LWBS rate.

\section{Abbreviations}

ED Emergency Department

LWBS Leaving Without Being Seen (by a physician)

KHCH Kanta-Häme Central Hospital

\section{Declarations}

Ethical approval and consent to participate

Ethics committee approval was waived according to the Finnish legislation on medical research. The study was a register-based study and entailed no contact with patients.

Consent for publication

Not applicable.

Availability of data and material

Coded data is available from the corresponding author on reasonable request.

Competing interests

The authors declare that they have no competing interests.

Funding

This study was funded by the Ministry of Social Affairs and Health through the research funds of KantaHäme Central Hospital and Tampere University Hospital as well as a grant from the Finnish Society of Emergency Medicine.

Authors' contributions 
AP and TI planned the study. MH analyzed the data with help of AP and TL. MH drafted the manuscript together with AP. AP was the responsible investigator. TI gathered the original data from the medical records with the help of TL. VH participated in analysis and drafting the manuscript. Finally, all authors read and approved the manuscript.

\section{Acknowledgements}

The authors would like to thank Mrs. Virginia Mattila for her valuable support for language editing during this research and RN Anu Pakkanen for support with applications. We would also like to thank the triage nurses and secretaries in $\mathrm{KHCH}$ ED for keeping the records and E-book in order.

\section{References}

1. Zocchi MS, McClelland MS, Pines JM. Increasing throughput: Results from a 42-hospital Collaborative to Improve Emergency Department Flow. Jt Comm J Qual Patient Saf. 2015;12:53242.

2. Sørup CM, Jacobsen P, Forberg JL. A literature review analyzing endorsed performance and qualityin-care measures for emergency department assessment. Scand J Trauma Resus. 2013;21:62.

3. Shaikh SB, Jerrard DA, Witting MD, Winters ME, Brodeur MN. How Long Are Patients Willing to Wait in the Emergency Department Before Leaving Without Being Seen? West J Emerg Med. 2012;13:463-7.

4. Carron PN, Yersin B, Trueb L, Gonin P, Hugli O. Missed Opportunities: Evolution of Patients Leaving without Being Seen or against Medical Advice during a Six-Year Period in a Swiss Tertiary Hospital Emergency Department. BioMed Res Int. 2014;2014:690368.

5. Rowe BH, Channan P, Bullard M, Blitz S, Saunders LD, Rosychuk RJ, et al. Characteristics of Patients Who Leave Emergency Departments without Being Seen. J AEM. 2006;13:848-52.

6. Rautava V-P, Palomäki E, Innamaa T, Perttu M, Lehto M, Palomäki A. Improvement in self-reported confidence in nurses' professional skills in the emergency department. Scand J Trauma Resusc Emerg Med 2013;21:16.

7. Heikkilä I, Kuusisto H, Stolberg A, Palomäki A. Stroke thrombolysis given by emergency physicians cuts in-hospital delays significantly immediately after implementing a new treatment protocol. Scand J Trauma Resusc Emerg Med. 2016;24:46.

8. Heikkilä I, Kuusisto H, Holmberg M, Palomäki A. Fast Protocol for Treating Acute Ischemic Stroke by Emergency Physicians. Ann Emerg Med. 2019;73:105-12.

9. Ojakäär A, Purdy M, Kechagias A, Järvelin U, Palomäki A. Immediate effects of urgent reorganisation of emergency department-based treatment pathway in nonperforated appendicitis: a retrospective study. BMC Emerg Med. 2020;20:45.

10. Tuominen J, Hällberg V, Oksala N, Palomäki A, Lukkarinen T, Roine A. NYU-EDA in modelling the effect of COVID-19 on patient volumes in a Finnish emergency department. BMC Emerg Med. 2020;20:97. 
11. Naskali J, Palomäki A, Harjola V-P, Hällberg V, Rautava V-P, Innamaa T. Emergency Medicine in Finland: First Year Experiences of Specialist Training. Acad J Emerg Med. 2014;13:26-9.

12. Lehtonen $H$, Lukkarinen $T$, Kämäräinen V, Rautava V-P, Parviainen P, Palomäki A. Improving Emergency Department Capacity Efficiency. Signa Vitae. 2016;12:52-7.

13. Kankaanpää M, Raitakari M, Muukkonen L, Gustafsson S, Heitto M, Palomäki A, Suojanen K, Harjola VP. Use of point-of-care testing and early assessment model reduces length of stay for ambulatory patients in an emergency department. Scand J Trauma Resusc Emerg Med. 2016;24:125.

14. Gilligan P, Joseph D, Winder S, Keeffe FO, Oladipo O, Ayodele T, et al. DNW--"did not wait" or "demographic needing work": a study of the profile of patients who did not wait to be seen in an Irish emergency department. Emerg Med J. 2009;26:780-2.

15. Grosgurin O, Cramer B, Schaller M, Sarasin FP, Rutschmann OT. Patients leaving the emergency department without being seen by a physician: a retrospective database analysis. Swiss Med Wkly. 2013;12:143.

16. Parekh KP, Russ S, Amsalem DA, Rambaran N, Wright SW. Who leaves the emergency department without being seen? A public hospital experience in Georgetown, Guyana. BMC Emerg Med. 2013;13:10.

17. Liu S, Nie H, Huang W, Liu X, Luo L, Lau WB, et al. Characteristics of patients who leave the emergency department without being seen: the first report in China. Emerg Med Australas. 2014;26:243-8.

18. Joshi AU, Randolph FT, Chang AM, Slovis BH, Rising KL, Sabonjian M, Sites FD, Hollander JE. Impact of Emergency Department Tele-intake on Left Without Being Seen and Throughput Metrics. Acad Emerg Med. 2020;27:139-47.

19. Rowe BH, McRae A, Rosychuk RJ. Temporal trends in emergency department volumes and crowding metrics in a western Canadian province: a population-based, administrative data study. BMC Health Serv Res. 2020;20:356.

20. Xu Y, Ho V. Freestanding emergency departments in Texas do not alleviate congestion in hospitalbased emergency departments. Am J Emerg Med. 2020:38;471-6

21. Lucas J, Batt RJ, Soremekun OA. Setting wait times to achieve targeted left-without-being-seen rates. Am J Emerg Med. 2014;32:342-5.

22. Sparrow N, Pestell T, Clancy M. Children who leave the Emergency Department without being seen: a Retrospective Case Note Review. Emerg Med J. 2015;32:712-5.

23. Fayyaz J, Khursheed M, Mir MU, Mehmood A. Missing the boat: odds for the patients who leave ED without being seen. BMC Emerg Med. 2013;13:1.

24. Baibergenova A, Leeb K, Jokovic A, Gushue S. Missed Opportunity: Patients Who Leave Emergency Departments without Being Seen. Healthc Policy. 2006;1:35-42.

25. Mohsin M, Young L, leraci S, Bauman AE. Factors associated with walkout of patients from New South Wales hospital emergency departments, Australia. Emerg Med Australas. 2005;17:434-42. 
26. Sun BC, Binstadt ES, Pelletier A, Camargo Jr CA. Characteristics and temporal trends of "left before being seen" visits in US emergency departments, 1995-2002. J Emerg Med. 2007;32:211-5.

27. Hitti E, Hadid D, Tamim H, Al Hariri M, El Sayed M. Left without being seen in a hybrid point of service collection model emergency department. Am J Emerg Med. 2020;38:497-502.

28. Arendt KW, Sadosty AT, Weaver AL, Brent CR, Boie ET. The left-without-being-seen patients: what would keep them from leaving? Ann Emerg Med . 2003;42:317-23.

29. Johnson M, Myers S, Wineholt J, Pollack M, Kusmiesz AL. Patients who leave the emergency department without being seen. J Emerg Nurs. 2009;35:105-8.

30. Pajunen P, Koukkunen H, Ketonen $M$, Jerkkola T, Immonen-Räihä P, Kärjä-Koskenkari P, Mähönen $M$, Niemelä M, Kuulasmaa K, Palomäki P, Mustonen J, Lehtonen A, Arstila M, Vuorenmaa T, Lehto S, Miettinen H, Torppa J, Tuomilehto J, Kesäniemi YA, Pyörälä K, Salomaa V. The validity of the Finnish Hospital Discharge Register and Causes of Death Register data on coronary heart disease. Eur $J$ Cardiovasc Prev Rehabil. 2005;12:132-7.

31. Chrusciel J, Fontaine X, Devillard A, Cordonnier A, Kanagaratnam L, Laplanche D, Sanchez S. Impact of the implementation of a fast-track on emergency department length of stay and quality of care indicators in the Champagne-Ardenne region: a before-after study. BMJ Open. 2019;9:e026200.

32. Jesionowski M, Riordan J, Quatrara B. Does a Provider in Triage and Rapid Medical Evaluation Help With Left Without Being Seen Rates and ED Crowding? J Emerg Nurs 2019;45:38-45.

33. Tlapa D, Zepeda-Lugo CA, Tortorella GL, Baez-Lopez YA, Limon-Romero J, Alvarado-Iniesta A, Rodriguez-Borbon MI. Effects of Lean Healthcare on Patient Flow: A Systematic Review. Value Health. 2020;23:260-73.

\section{Figures}


$1,6 \%$

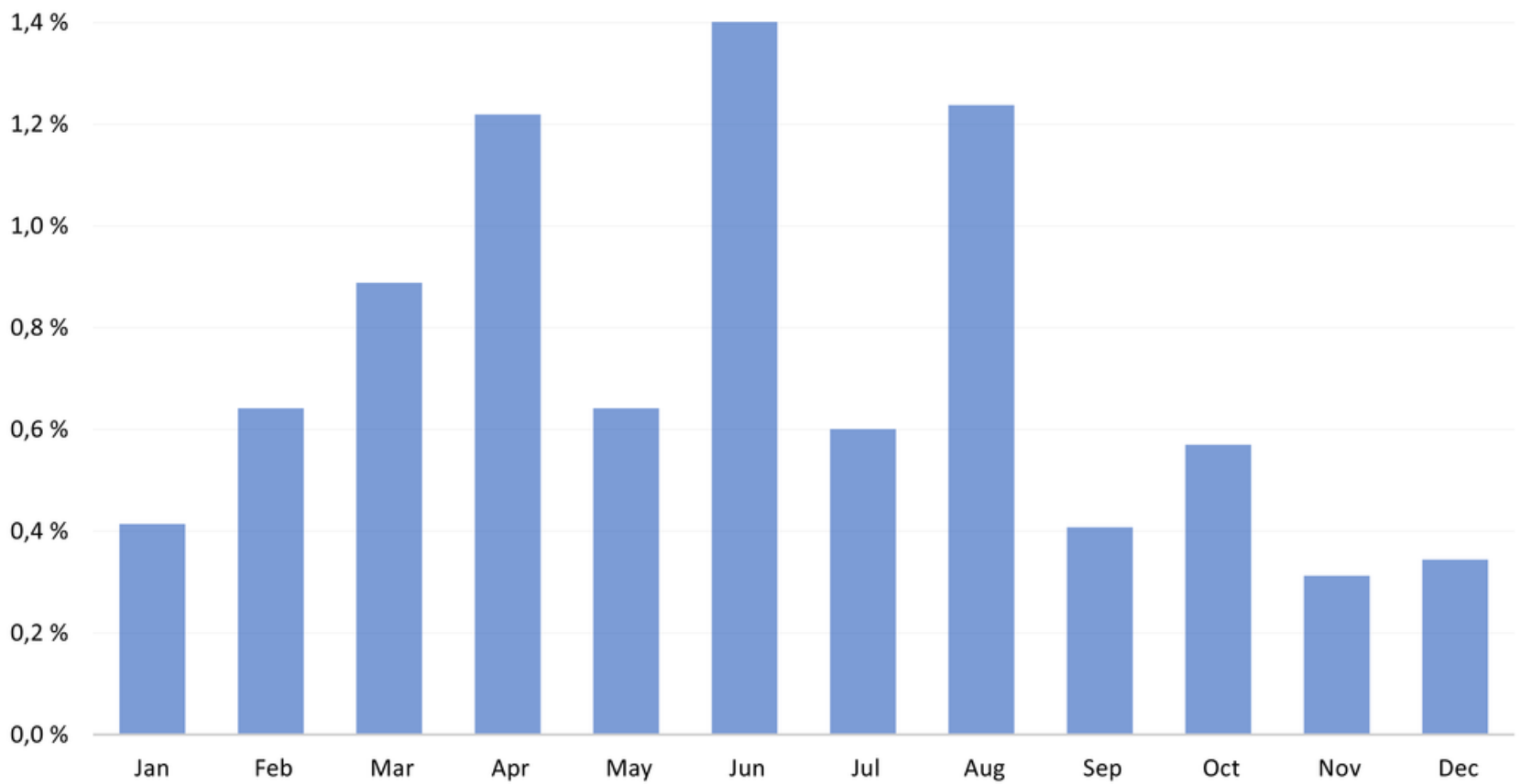

Figure 1

Monthly rate in LWBS patients of all ED patients as percentages. 


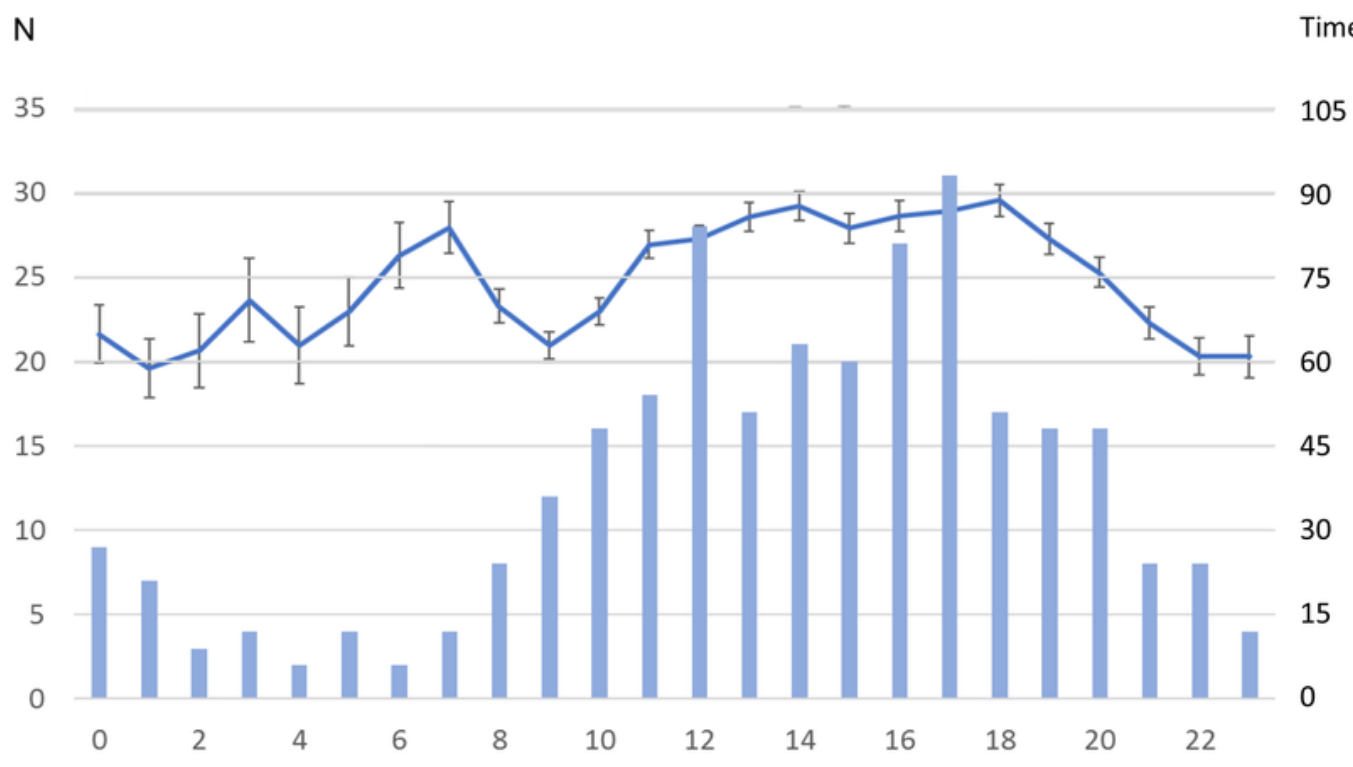

Time (min)

90

75

60

30

15

A

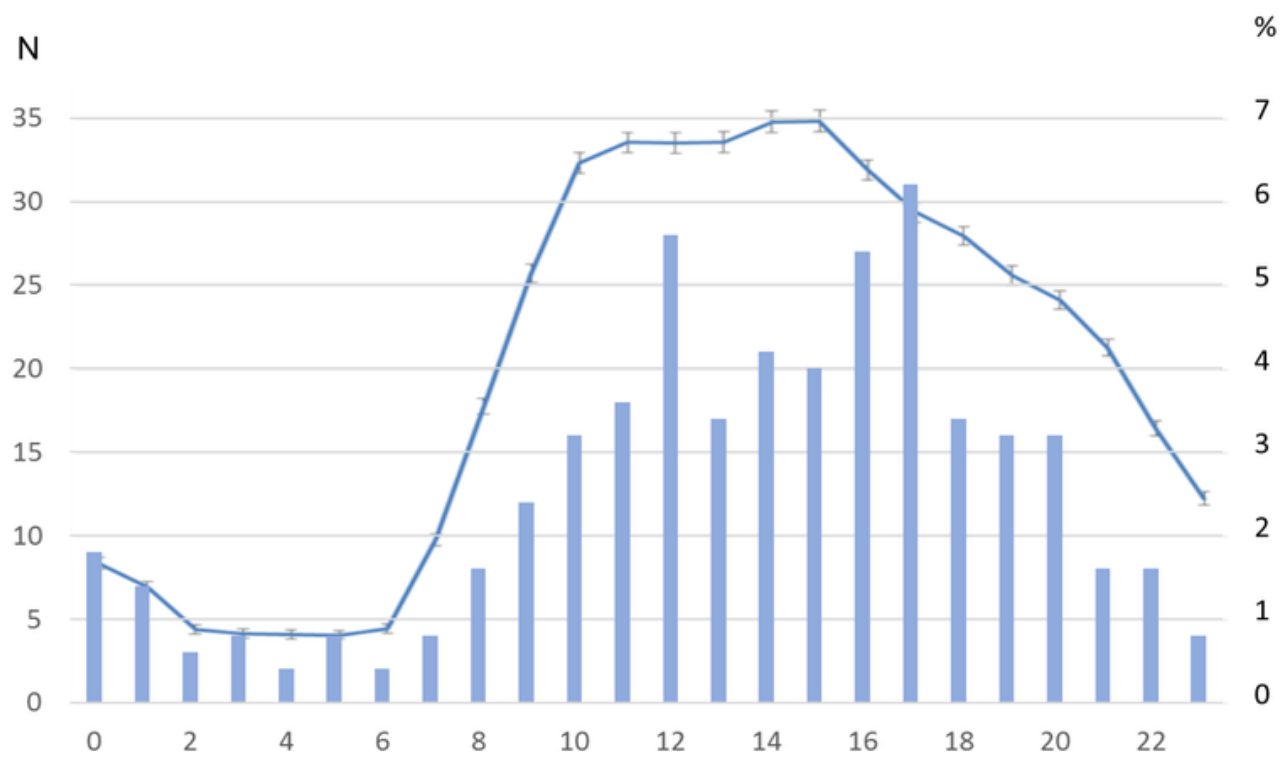

B

Figure 2

a. Hourly numbers of LWBS patients over 12 months presented as pillars (left Y-axis) and hourly distribution of all ED patient visits in percentages as the blue line (mean, $95 \% \mathrm{Cl}$, right Y-axis). b. Hourly numbers of LWBS patients over 12 months presented as pillars (left Y-axis) and hourly waiting times of all visits before seeing a doctor shown by the hour as the blue line (mean, $95 \% \mathrm{Cl}$, right $\mathrm{Y}$-axis). 